\title{
SOLUSI POSITIF DARI PERSAMAAN LEONTIEF DISKRIT
}

\author{
RASITA ANAS \\ Program Studi Matematika, \\ Fakultas Matematika dan Ilmu Pengetahuan Alam, Universitas Andalas, \\ Kampus UNAND Limau Manis Padang, Indonesia, \\ rasitaanas@rocketmail.com
}

\begin{abstract}
Abstrak. Pada tulisan ini akan diuraikan tentang bagaimanakah solusi dari persamaan Leontief diskrit dengan menggunakan invers Drazin dan sistem singular diskrit. Untuk $C$ singular sistem $C \mathbf{x}_{n+1}=(I-L+C) \mathbf{x}_{n}-\mathbf{d}_{n}$, tidak mempunyai solusi. Hal ini disebabkan adanya kondisi awal yang tidak dapat memberikan solusi untuk sistem. Kondisi awal yang dapat memberikan solusi untuk sistem disebut sebagai kondisi awal yang konsisten [4]. Perlu diperhatikan bahwa solusi $\mathbf{x}_{n}$ untuk sistem mungkin positif atau mungkin saja non positif. Solusi $\mathbf{x}_{n}$ dikatakan positif jika $x_{i n}>0$ untuk setiap $i=1,2, \cdots, n$ dan dikatakan non positif jika $x_{i n} \leq 0$ untuk setiap $i=1,2, \cdots, n$. Jika solusi $\mathbf{x}_{n}$ untuk sistem adalah positif maka $\mathbf{x}_{n}$ dikatakan solusi positif dari persamaan Leontief. Dengan Teorema yang diberikan, diperoleh syarat cukup untuk kepositifan dari solusi persamaan Leontief diskrit.
\end{abstract}

Kata Kunci: Invers Drazin, persamaan Leontief, sistem singular diskrit

\section{Pendahuluan}

Diberikan suatu sistem persamaan beda linier (linear difference equation) sebagai berikut:

$$
C \mathbf{x}_{n+1}=(I-L+C) \mathbf{x}_{n}-\mathbf{d}_{n}, \quad n \in \mathbb{Z}_{+},
$$

di mana $I \in \mathbb{R}^{r \times r}$ adalah matriks identitas, $C, L \in \mathbb{R}^{r \times r}$ dan $\mathbf{d}_{n}, \mathbf{x}_{n} \in \mathbb{R}^{r}$. Sistem di atas dapat ditulis sebagai:

$$
C \mathbf{x}_{n+1}=E \mathbf{x}_{n}-\mathbf{d}_{n}, \quad n \in \mathbb{Z}_{+}
$$

di mana $E=(I-L+C)$. Notasi $\mathbb{R}^{r \times r}$ menyatakan himpunan matriks-matriks riil berukuran $r \times r, \mathbb{R}_{+}^{r \times r}$ menyatakan himpunan matrik-matriks riil berukuran $r \times r$ yang entri-entrinya non negatif, $\mathbb{R}_{-}^{r \times r}$ menyatakan himpunan matrik-matriks riil berukuran $r \times r$ yang entri-entrinya non positif, $\mathbb{R}_{++}^{r \times r}$ menyatakan himpunan matriks-matriks riil berukuran $r \times r$ di mana entri-entrinya adalah bilangan riil positif, $\mathbb{R}^{r}$ menyatakan himpunan vektor berdimensi $r, \mathbb{Z}_{+}$menyatakan himpunan bilangan bulat non negatif, dan $\mathbb{C}$ menyatakan himpunan bilangan kompleks.

Jika matriks $L=\left[l_{i j}\right]$ memenuhi:

$$
\begin{aligned}
& 0 \leq l_{i j} \leq 1, \quad 1 \leq i, j \leq r, \\
& \sum_{i=1}^{r} l_{i j} \leq 1, \quad 1 \leq j \leq r .
\end{aligned}
$$


Maka persamaan (1.1) disebut sebagai persamaan Leontief.

Jika $C$ adalah matriks non singular, maka solusi dari sistem (1.1) adalah :

$$
\mathbf{x}_{n}=C^{-1}\left[(I-L+C)^{n} \mathbf{x}_{0}+\sum_{i=0}^{n-1}(I-L+C)^{n-i-1} \mathbf{d}_{i}\right]
$$

untuk suatu vektor $\mathbf{v} \in \mathbb{R}$.

Untuk $C$ singular, sistem (1.1) mungkin tidak mempunyai solusi. Hal ini disebabkan adanya kondisi awal yang tidak dapat memberikan solusi untuk sistem (1.1). Kondisi awal yang dapat memberikan solusi untuk sistem (1.1) disebut sebagai kondisi awal yang konsisten.

Perlu diperhatikan bahwa solusi $\mathbf{x}_{n}$ untuk sistem (1.1) mungkin positif atau mungkin saja non positif. Solusi $\mathbf{x}_{n}$ dikatakan positif jika $x_{i n}>0$ untuk setiap $i=1,2, \cdots, n$ dan dikatakan negatif jika $x_{i n}<0$ untuk setiap $i=1,2, \cdots, n$. Jika solusi $\mathbf{x}_{n}$ untuk sistem (1.1) adalah positif maka $\mathbf{x}_{n}$ dikatakan solusi positif dari persamaan Leontief.

\section{Solusi Positif dari Persamaan Leontief Diskrit}

Asumsikan bahwa matriks $C$ adalah singular dan $\operatorname{det}(\lambda C-E) \neq 0$ untuk suatu $\lambda \in \mathbb{C}$, maka ada $\lambda \in \mathbb{C}$ sedemikian sehingga $(\lambda C-E)^{-1}$ ada. Dengan mengalikan sistem (1.1) dengan $(\lambda C-E)^{-1}$, maka

$$
\begin{aligned}
(\lambda C-E)^{-1} C \mathbf{x}_{n+1} & =(\lambda C-E)^{-1} E \mathbf{x}_{n}-(\lambda C-E)^{-1} \mathbf{d}_{n} \\
\widehat{C} \mathbf{x}_{n+1} & =\widehat{E} \mathbf{x}_{n}-\widehat{\mathbf{d}}_{n}
\end{aligned}
$$

dengan $\widehat{C}=(\lambda C-E)^{-1} C, \widehat{E}=(\lambda C-E)^{-1} E, \widehat{\mathbf{d}}_{n}=(\lambda C-E)^{-1} \mathbf{d}_{n}$.

Untuk $\lambda=1$, pada sistem

$$
C \mathbf{x}_{n+1}=(I-L+C) \mathbf{x}_{n}
$$

berlaku $\lambda C-(C+I-L)=(\lambda-1) C+L-1=(L-I)$. Karena $(I-l)^{-1}$ ada maka $(\lambda C-(C+I-L))^{-1}$ ada. Selanjutnya, misalkan

$$
\begin{aligned}
\widehat{C} & =(\lambda C-E)^{-1} C \\
& =(\lambda C-(C+I-L))^{-1} C \\
& =(-I+L)^{-1} C \\
& =-(I-L)^{-1} C \\
\widehat{E} & =(\lambda C-E)^{-1} E \\
& =(\lambda C-(C+I-L))^{-1}(C+I-L) \\
& =-(I-L)^{-1}(I-L+C) \\
& =-I-(I-L)^{-1} C \\
& =\widehat{C}-I .
\end{aligned}
$$


Solusi umum dari (2.1) diberikan oleh:

$$
\begin{aligned}
\mathbf{x}_{n} & =\left(\widehat{C}^{D}(\widehat{C}-I)\right)^{n} \widehat{C} \widehat{C}^{D} \mathbf{x}_{0}-\widehat{C}^{D} \sum_{j=0}^{n-1}\left(\widehat{C}^{D}(\widehat{C}-I)\right)^{n-j-1}(I-L)^{-1} \mathbf{d}_{j} \\
& +\left(I-\widehat{C} \widehat{C}^{D}\right) \sum_{i=0}^{k-1}\left(\widehat{C}(\widehat{C}-I)^{D}\right)^{i}(\widehat{C}-I)^{D}(I-L)^{-1} \mathbf{d}_{n+i}, \quad n \geq 1
\end{aligned}
$$

$\mathbf{x}_{n}$ pada persamaan (2.3) adalah solusi dari persamaan (2.1).

Selanjutnya akan ditunjukkan bahwa $\mathbf{x}_{n}$ tak negatif, untuk $n=1,2, \cdots, N$. Teorema berikut merupakan syarat cukup untuk kepositifan dari solusi persamaan Leontief diskrit.

Teorema 2.1. [3] Misalkan terdapat matriks $C, L, I \in \mathbb{R}^{r \times r}$ sedemikian sehingga

(i) Semua elemen diagonal dari $\widehat{C}$ tidak nol dan tidak sama dengan satu.

(ii) Semua elemen diagonal dari $\widehat{C}^{D}$ adalah tidak nol.

(iii) $\widehat{C} \preceq 0, \widehat{C}^{D} \preceq 0$ dan $\widehat{E} \preceq 0$.

(iv) $\widehat{\boldsymbol{d}}_{i} \preceq \boldsymbol{O}$ dan $\boldsymbol{x}_{0} \succeq \boldsymbol{0}$.

Misalkan juga

$$
\boldsymbol{x}_{n}=L_{n}\left(\boldsymbol{x}_{0}\right)+\boldsymbol{z}_{k}(n)
$$

di mana

$$
L_{n}\left(\boldsymbol{x}_{0}\right)=\left(\widehat{C}^{D} \widehat{E}\right)^{n} \widehat{C} \widehat{C}^{D} \boldsymbol{x}_{0}+\widehat{C}^{D} \sum_{i=0}^{n-1}\left(\widehat{C}^{D} \widehat{E}\right)^{n-i-1} \widehat{\boldsymbol{d}}_{i}
$$

dan

$$
\boldsymbol{z}_{k}(n)=-\left(I-\widehat{C} \widehat{C}^{D}\right) \sum_{i=0}^{k-1}\left(\widehat{C} \widehat{E}^{D}\right)^{i} \widehat{E}^{D} \widehat{\boldsymbol{d}}_{n+i}
$$

Maka $\boldsymbol{x}_{n}>\boldsymbol{O}$ untuk $1 \leq n \leq N$ dan vektor $\boldsymbol{x}_{0}$ memenuhi $\boldsymbol{x}_{0} \succeq \frac{\left(I-\widehat{C} \widehat{C}^{D}\right) \sum_{i=0}^{k-1}\left(\widehat{C} \widehat{E}^{D}\right)^{i}\left(\widehat{E}^{D}\right)\left(\widehat{\boldsymbol{d}}_{n+i}\right)+\sum_{i=0}^{n-1}\left[\left(\widehat{C}^{D}\right)_{\min }\right]^{n-i}\left[(\widehat{E})_{\min }\right]^{n-i-1} r^{n-i+1} \widehat{\boldsymbol{d}}_{i}}{\left(d_{\max }\left(\widehat{C}^{D}\right)\right)^{n+1}\left(d_{\max }(\widehat{E})\right)^{n} d_{\max }(\widehat{C})}$.

Bukti. $\mathbf{x}_{n}>\mathbf{0}$ jika dan hanya jika $L_{n}\left(\mathbf{x}_{0}\right)+\mathbf{z}_{k}(n) \succeq \mathbf{0}$, yaitu

$$
\left(\widehat{C}^{D} \widehat{E}\right)^{n} \widehat{C} \widehat{C}^{D} \mathbf{x}_{0} \succeq-\widehat{A}^{D} \sum_{i=0}^{n-1}\left(\widehat{C}^{D} \widehat{E}\right)^{n-i-1} \widehat{\mathbf{d}}_{i}-\mathbf{z}_{k}(n) .
$$

Dari [3], diperoleh bahwa $\widehat{C} \preceq 0$ dan $\widehat{C}^{D} \preceq 0$, sehingga

$$
\widehat{C} \widehat{C}^{D} \succeq d_{\max }(\widehat{C}) d_{\max }\left(\widehat{C}^{D}\right) I .
$$

Selain itu karena $\mathbf{x}_{0} \succeq \mathbf{0}$, maka diperoleh

$$
\widehat{C} \widehat{C}^{D} \mathbf{x}_{0} \succeq d_{\max }(\widehat{C}) d_{\max }\left(\widehat{C}^{D}\right) \mathbf{x}_{0} .
$$

Dari [3] diperoleh bahwa $\widehat{C} \preceq 0$ dan $\widehat{E} \preceq 0$, sehingga

$$
\left(\widehat{C}^{D} \widehat{E}\right)^{n} \succeq\left(d_{\max }\left(\widehat{C}^{D}\right)\right)^{n}\left(d_{\max }(\widehat{E})\right)^{n} I .
$$


Dari (2.8) dan (2.9) diperoleh

$$
\left(\widehat{C}^{D} \widehat{E}\right)^{n} \widehat{C} \widehat{C}^{D} \boldsymbol{x}_{0} \succeq\left(d_{\max }\left(\widehat{C}^{D}\right)\right)^{n+1}\left(d_{\max }(\widehat{E})\right)^{n} d_{\max }(\widehat{C}) \boldsymbol{x}_{0} .
$$

Berdasarkan [5] diperoleh

$$
\begin{aligned}
0 & \preceq\left(\widehat{C}^{D} \widehat{E}\right)^{n-i-1} \\
& \preceq\left(\left(-\widehat{C}^{D}\right)(-\widehat{E})\right)^{n-i-1} \\
& \left.\preceq\left(-\widehat{C}^{D}\right)_{\max }(-\widehat{E})_{\max }\right)^{n-i-1} r^{n-i-1+1} \\
& \preceq\left[\left(-\widehat{C}^{D}\right)_{\max }(-\widehat{E})_{\max }\right]^{n-i-1} r^{n-i} .
\end{aligned}
$$

Karena $-\widehat{C}^{D} \succeq 0$, maka (2.11) menjadi

$$
\begin{aligned}
\left(-\widehat{C}^{D}\right)\left(\widehat{C}^{D} \widehat{E}\right)^{n-i-1} & \preceq r\left(-\widehat{C}^{D}\right)_{\max }\left(\widehat{C}^{D} \widehat{E}\right)_{\max }^{n-i-1} \\
& \preceq r\left(-\widehat{C}^{D}\right)_{\max }\left[\left(-\widehat{C}^{D}\right)_{\max }(-\widehat{E})_{\max }\right]^{n-i-1} r^{n-i} \\
& =\left[\left(-\widehat{C}^{D}\right)_{\max }\right]^{n-i}\left[(-\widehat{E})_{\max }\right]^{n-i-1} r^{n-i+1} .
\end{aligned}
$$

Karena $\widehat{\mathbf{d}}_{i} \preceq \mathbf{0}$, maka (2.12) menjadi

$$
\left(-\widehat{C}^{D}\right)\left(\widehat{C}^{D} \widehat{E}\right)^{n-i-1} \widehat{\mathbf{d}}_{i} \succeq\left[\left(-\widehat{C}^{D}\right)_{\max }\right]^{n-i}\left[(-\widehat{E})_{\max }\right]^{n-i-1} r^{n-i+1} \widehat{\mathbf{d}}_{i} .
$$

Oleh karena itu

$$
\left(-\widehat{C}^{D}\right) \sum_{i=0}^{n-1}\left(\widehat{C}^{D} \widehat{E}\right)^{n-i-1} \widehat{\mathbf{d}}_{i} \succeq \sum_{i=0}^{n-1}\left[\left(-\widehat{C}^{D}\right)_{\max }\right]^{n-i}\left[(-\widehat{E})_{\max }\right]^{n-i-1} r^{n-i+1} \widehat{\mathbf{d}}_{i} .
$$

Karena $\left(-\widehat{C}^{D}\right)_{\max }=-\left(\widehat{C}^{D}\right)_{\min }$ dan $(-\widehat{E})_{\max }=-(\widehat{E})_{\min }$, maka $(2.14)$ menjadi

$$
\left(-\widehat{C}^{D}\right) \sum_{i=0}^{n-1}\left(\widehat{C}^{D} \widehat{E}\right)^{n-i-1} \widehat{\mathbf{d}}_{i} \succeq-\sum_{i=0}^{n-1}\left[\left(\widehat{C}^{D}\right)_{\min }\right]^{n-i}\left[(\widehat{E})_{\min }\right]^{n-i-1} r^{n-i+1} \widehat{\mathbf{d}}_{i} .
$$

Dari (2.10) dan (2.15) maka disimpulkan bahwa (2.6) memenuhi

$$
\begin{aligned}
\left(d_{\max }\left(\widehat{C}^{D}\right)\right)^{n+1}\left(d_{\max }(\widehat{E})\right)^{n} d_{\max }(\widehat{C}) \boldsymbol{x}_{0} \preceq & -\sum_{i=0}^{n-1}\left[\left(\widehat{C}^{D}\right)_{\min }\right]^{n-i}\left[(\widehat{E})_{\min }\right]^{n-i-1} \widehat{\boldsymbol{d}}_{i} r^{n-i+1} \\
& +-\left(I-\widehat{C} \widehat{C}^{D}\right) \sum_{i=0}^{k-1}\left(\widehat{C}^{D} \widehat{C}^{D}\right)^{i} \widehat{E}^{D} \widehat{\mathbf{d}}_{n+i}
\end{aligned}
$$

atau dapat ditulis,

$$
\mathbf{x}_{0} \succeq \frac{\left(I-\widehat{C} \widehat{C}^{D}\right) \sum_{i=0}^{k-1}\left(\widehat{C} \widehat{E}^{D}\right)^{i}\left(\widehat{E}^{D}\right)\left(\widehat{\mathbf{d}}_{n+i}\right)+\sum_{i=0}^{n-1}\left[\left(\widehat{C}^{D}\right)_{\min }\right]^{n-i}\left[(\widehat{E})_{\min }\right]^{n-i-1} r^{n-i+1} \widehat{\mathbf{d}}_{i}}{\left(d_{\max }\left(\widehat{C}^{D}\right)\right)^{n+1}\left(d_{\max }(\widehat{E})\right)^{n} d_{\max }(\widehat{C})}
$$

Contoh. Misalkan diberikan suatu sistem

$$
C \mathbf{x}_{n+1}=E \mathbf{x}_{n}-\mathbf{d}_{n}
$$

di mana,

$$
L=\left(\begin{array}{lll}
0.3 & 0.3 & 0.3 \\
0.4 & 0.1 & 0.5 \\
0.3 & 0.5 & 0.2
\end{array}\right), C=\left(\begin{array}{ccc}
0.3 & 0.4 & 0.45 \\
0 & 0 & 0 \\
0.6 & 0.8 & 0.9
\end{array}\right), \mathbf{d}_{n}=\left(\begin{array}{c}
18+2 n \\
32+3 n \\
57+n
\end{array}\right)
$$


Akan ditentukan vektor $\mathbf{x}_{0}$, sedemikian sehingga sistem tersebut adalah positif. Perhatikan bahwa,

$$
\begin{aligned}
\widehat{C} & =-(I-L)^{-1} C \\
& =\left(\begin{array}{ccc}
-8.3617 & -11.149 & -12.543 \\
-9 & -12 & -13.5 \\
-9.5106 & -12.681 & -14.266
\end{array}\right)
\end{aligned}
$$

maka

$$
\widehat{C}^{D}=\left(\begin{array}{l}
-6.9732 \times 10^{-3}-9.2977 \times 10^{-3}-1.0460 \times 10^{-2} \\
-7.5055 \times 10^{-3}-1.0007 \times 10^{-2}-1.1258 \times 10^{-2} \\
-7.9313 \times 10^{-3}-1.0575 \times 10^{-2}-1.1897 \times 10^{-2}
\end{array}\right)
$$

dan

$$
\begin{aligned}
(\widehat{E}) & =(\widehat{C}-I) \\
& =\left(\begin{array}{ccc}
-9.3617 & -11.149 & -12.543 \\
-9 & -13 & -13.5 \\
-9.5106 & -12.681 & -15.266
\end{array}\right)
\end{aligned}
$$

sehingga diperoleh

$$
(\widehat{E})^{D}=\left(\begin{array}{c}
-6.794 \times 10^{-3}-9.0295 \times 10^{-3}-1.0158 \times 10^{-2} \\
-7.2889 \times 10^{-3}-9.7406 \times 10^{-3}-1.0933 \times 10^{-2} \\
-7.7024 \times 10^{-3}-1.0270 \times 10^{-2}-1.1576 \times 10^{-2}
\end{array}\right) .
$$

Selanjutnya,

$$
\begin{aligned}
\widehat{\mathbf{d}}_{n} & =-(I-L)^{-1} \mathbf{d}_{n} \\
& =\left(\begin{array}{c}
-53.830 n-954.90 \\
-60.0 n-1070.0 \\
-58.936 n-1098.1
\end{array}\right) .
\end{aligned}
$$

Untuk $n=1$, diperoleh

$$
\begin{aligned}
\mathbf{x}_{0} & \succeq \frac{\left(I-\widehat{C} \widehat{C}^{D}\right)\left(\widehat{C} \widehat{E}^{D}\right)^{0} \widehat{E}^{D} \widehat{d}_{1}+\left[\left(\widehat{C}^{D}\right)_{\min }\right]\left[(\widehat{E})_{\min }\right]^{0} r^{2} \widehat{d}_{0}}{\left(d_{\max }\left(\widehat{C}^{D}\right)\right)^{2}\left(d_{\max }(\widehat{E})\right) d_{\max }(\widehat{C})} \\
& \succeq\left(\begin{array}{l}
26861 \\
30116 \\
30891
\end{array}\right)
\end{aligned}
$$

Misalkan $\mathbf{x}_{0}=\left(\begin{array}{l}26861 \\ 30116 \\ 30891\end{array}\right)$. Maka solusi untuk $\mathbf{x}_{n}$ di mana $n=1,2,3,4$ adalah

$$
\mathbf{x}_{1}=\left(\begin{array}{l}
28187 \\
30338 \\
32060
\end{array}\right), \mathbf{x}_{2}=\left(\begin{array}{l}
29030 \\
31245 \\
33018
\end{array}\right), \mathbf{x}_{3}=\left(\begin{array}{l}
29897 \\
32179 \\
34005
\end{array}\right), \mathbf{x}_{4}=\left(\begin{array}{l}
30756 \\
33103 \\
34982
\end{array}\right)
$$


Misalkan $\mathbf{x}_{0}=\left(\begin{array}{l}36861 \\ 40116 \\ 42891\end{array}\right)$. Maka solusi untuk $\mathbf{x}_{n}$ dengan $n=1,2,3,4$ adalah

$$
\mathbf{x}_{1}\left(\begin{array}{c}
38455 \\
41390 \\
43739
\end{array}\right), \mathbf{x}_{2}=\left(\begin{array}{l}
39594 \\
42615 \\
45034
\end{array}\right), \mathbf{x}_{3}=\left(\begin{array}{l}
40766 \\
43877 \\
46367
\end{array}\right), \mathbf{x}_{4}=\left(\begin{array}{l}
39189 \\
42181 \\
44574
\end{array}\right) \text {. }
$$

\section{Kesimpulan}

Solusi $\mathbf{x}_{n}$ dari sistem persamaan Leontief diskrit

$$
C \mathbf{x}_{n+1}=E \mathbf{x}_{n}-\mathbf{d}_{n}, n \in \mathbb{Z}_{+}
$$

adalah positif jika nilai $\mathbf{x}_{0}$ memenuhi,

$\mathbf{x}_{0} \succeq \frac{\left(I-\widehat{C} \widehat{C}^{D}\right) \sum_{i=0}^{k-1}\left(\widehat{C} \widehat{E}^{D}\right)^{i}\left(\widehat{E}^{D}\right)\left(\widehat{\mathbf{d}}_{n+i}\right)+\sum_{i=0}^{n-1}\left[\left(\widehat{C}^{D}\right)_{\min }\right]^{n-i}\left[(\widehat{E})_{\min }\right]^{n-i-1} r^{n-i+1} \widehat{\mathbf{d}}_{i}}{\left(d_{\max }\left(\widehat{C}^{D}\right)\right)^{n+1}\left(d_{\max }(\widehat{E})\right)^{n} d_{\max }(\widehat{C})}$, asalkan

(i) Semua elemen diagonal dari $\widehat{C}$ tidak nol dan tidak sama dengan satu.

(ii) Semua elemen diagonal dari $\widehat{C}^{D}$ adalah tidak nol.

(iii) $\widehat{C} \preceq 0, \widehat{C}^{D} \preceq 0$ dan $\widehat{E} \preceq 0$.

(iv) $\widehat{\mathbf{d}}_{i} \preceq \mathbf{0}$ dan $\mathbf{x}_{0} \succeq \mathbf{0}$.

\section{Ucapan Terima kasih}

Penulis mengucapkan terima kasih kepada Bapak Dr. Muhafzan, Bapak Zulakmal, M.Si, Bapak Drs. Syafruddin M.Si dan Bapak Narwen, M.Si yang telah memberikan masukan dan saran sehingga paper ini dapat diselesaikan dengan baik.

\section{Daftar Pustaka}

[1] Anton, H. dan C. Rorres. 1991. Aljabar Linier Elementer Edisi 8. Jilid 1. Erlangga. Jakarta.

[2] Campbell, S.L. 1979. Generalized Inverses of Linear Transformations. Dover. New York.

[3] Jodar, L dan Merello, P. 2010. Positive Solution of Discrete Dynamic Leontief Input-Output Model with Possibly Singular Capital Matrix. Mathematical and Computer Modelling. 52 : 1081 - 1087.

[4] Kaczorek, T. 1992. Linear Control Systems Volume 1. Research Studies Press LTD, England.

[5] R. Bellman. 1970. Introduction to Matriks Analysis. McGraw-Hill. New York. 